\title{
Efficiently Compressible Density Operators via Entropy Maximization ${ }^{+}$
}

\author{
Serena Di Giorgio ${ }^{1,2, *}$ and Paulo Mateus ${ }^{1,2}$ (1) \\ 1 Department of Mathematics, Instituto Superior Técnico, Universidade de Lisboa, 1049-001 Lisboa, Portugal \\ 2 Instituto de Telecomunicações, 1049-001 Lisboa, Portugal; pmat@math.tecnico.ulisboa.pt \\ * Correspondence: digiorgio.srn@gmail.com; Tel.: +39-3472928285 \\ + Presented at the 11th Italian Quantum Information Science conference (IQIS2018), Catania, Italy, \\ 17-20 September 2018.
}

Received: 14 November 2019; Accepted: 9 May 2019; Published: 2 August 2019

\begin{abstract}
We address the problem of efficiently and effectively compress density operators (DOs), by providing an efficient procedure for learning the most likely $D O$, given a chosen set of partial information. We explore, in the context of quantum information theory, the generalisation of the maximum entropy estimator for DOs, when the direct dependencies between the subsystems are provided. As a preliminary analysis, we restrict the problem to tripartite systems when two marginals are known. When the marginals are compatible with the existence of a quantum Markov chain (QMC) we show that there exists a recovery procedure for the maximum entropy estimator, and moreover, that for these states many well-known classical results follow. Furthermore, we notice that, contrary to the classical case, two marginals, compatible with some tripartite state, might not be compatible with a QMC. Finally, we provide a new characterisation of quantum conditional independence in light of maximum entropy updating. At this level, all the Hilbert spaces are considered finite dimensional.
\end{abstract}

Keywords: maximum entropy density operators; quantum conditional independence; density operators recovery

\section{Introduction}

Quantum tomography allows to reconstruct a unique quantum state by performing a complete set of measurements on multiple copies of a quantum system. The amount of necessary resources to perform the reconstruction increases exponentially with the dimension of the system, and so, machine learning techniques provide more efficient procedures to infer the most likely quantum state from a chosen set of partial information.

When dealing with partial information, it is reasonable to choose a maximally non-committal estimator, with regard to the missing information, that coincides with the maximum (Shannon) entropy probability distribution (PD), as addressed by Jaynes [1] in the classical scenario. Since DOs generalise PDs, we analyse density operators that, spanning on the whole Hilbert space, maximise the von Neumann entropy. Concretely, we focus on the case of DOs for which the bipartite marginals are known.

By limiting our knowledge to the "first neighbour" interactions, we expect to obtain an exponential speedup concerning inference of the optimal DO. This is a well-known result for the analogous classical problem using PDs (see, for instance, [2,3]): given a set of random variables, ranged on finite domains, for which the given direct dependencies are tree-structured, Bayesian networks provide an optimal efficient learning procedure of the joint distribution. It turns out that the inferred joint always maximises the Shannon entropy from the provided bipartite marginals. Moreover, via the Chow-Liu learning algorithm [4], the closest tree to any graphical structure can be determined. The full joint PD is 
factorizable via Bayes rule [3], and thanks to the Markov condition [3], the factors depend merely on bipartite correlations.

Therefore, given a multipartite quantum system, the problem of inferring the DO that maximises the von Neumann entropy from its set of bipartite marginals is expected to be efficiently solvable, at least when it coincides with a purely classical tree. However, the following open question arises: is there a larger subset of DOs for which this result also holds? It is reasonable to begin our analysis with quantum trees, in particular, with the easiest non-trivial one-a tripartite system. Concretely, we consider a quantum system $\{A, B, C\}$ for which two of its bipartite marginals are known, say $\rho_{A B}$ and $\rho_{B C}$, and we ask whether there exists an efficient procedure for inferring, among all that have the same marginals, the tripartite quantum state that maximises the von Neumann entropy.

For our analysis, it is quite relevant to consider the results about quantum Markovianity, in particular, the definition of quantum Markov chain via CPTP maps composition stated in [5] and its entropic characterisation, known as quantum conditional independence [6,7]. We also benefitted from the results in [5,8,9] about recovery maps, that are summarised in [10], namely the operatorial versions of the strong subadditivity in [11,12] and the results about quantum Markov chains by Petz [7,13].

Interestingly, we find out that, differently from the classical scenario, the resolvability of the problem strictly depends on the nature of the given marginals. Indeed, we provide a necessary and sufficient condition to determine whether the pair of provided marginals allows for a concrete algebraic solution of the von Neumann entropy maximisation problem, and for that case, we explicitly give the analytic expression of the recovered tripartite density operator. Finally, we stress that by studying the conditional independence at the light of von Neumann entropy maximisation allowed to introduce a novel characterisation of quantum Markov chains.

\section{Results}

We start by stating the simplest recovery problem for tripartite systems, for that, we need to introduce the concept of compatibility for quantum states. We say that a set of marginal quantum states $\rho_{A B}, \rho_{B C}$ are compatible if there exists a tripartite state $\rho_{A B C}$ such that $\operatorname{Tr}_{A}\left(\rho_{A B C}\right)=\rho_{B C}$ and $\operatorname{Tr}_{C}\left(\rho_{A B C}\right)=\rho_{B C}$.

Problem 1 (Maximum Entropy Estimator). Given two compatible bipartite marginal quantum states $\rho_{A B} \in$ $\mathcal{L}\left(\mathcal{H}_{A B}\right), \rho_{B C} \in \mathcal{L}\left(\mathcal{H}_{B C}\right)$ finding the correspondent tripartite quantum state $\rho_{A B C} \in \mathcal{L}\left(\mathcal{H}_{A B C}\right)$ with highest von-Neumann entropy.

The solution for the above problem is given by

$$
\tilde{\rho}_{A B C}=\frac{1}{Z_{A B C}} \exp \left(\sum_{i j} \lambda_{i j} \Lambda_{i}^{(A)} \Lambda_{j}^{(B)} \mathbb{I}_{C}+\sum_{i j} \eta_{i j} \mathbb{I}_{A} \Lambda_{i}^{(B)} \Lambda_{j}^{(C)}\right)
$$

where $Z_{A B C}$ is a constant that enforces trace one on $\tilde{\rho}_{A B C} ; \lambda_{i j}$ and $\eta_{i j}$ are Lagrange multipliers which are constrained by the given partial traces; $\left\{\Lambda_{i}^{(X)}: i=0 \ldots n^{2}-1\right\}$ is a family of traceless, Hermitian matrices (plus the identity) that generate the Lie Algebra of $\mathcal{H}_{X}$ for $X=A, B, C$.

Given that this problem is overwhelming in general, we focus our attention on a well-behaved class of tripartite states. We recall that a tripartite state $\rho_{A B C}$ on $A \otimes B \otimes C$ is said to be a (quantum) Markov chain in order $A \leftrightarrow B \leftrightarrow C$ if there exists a recovery map $\mathcal{R}_{B \rightarrow B C}$ such that

$$
\rho_{A B C}=\mathcal{I}_{A} \otimes \mathcal{R}_{B \rightarrow B C}\left(\rho_{A B}\right),
$$

where a recovery map is an arbitrary trace-preserving completely positive map. Interestingly, there is an information-theoretic characterization for QMC based on conditional von Neumann entropy, which can be found, for instance, in [6]. Concretely, a tripartite state is a QMC iff $I_{\rho}(A: C \mid B)=0$, i.e., if the strong sub-additivity of von Neumann entropy [14] holds with equality. 
Our first result states that there exists an algebraic solution to Equation (1) when the provided marginals are compatible with a quantum Markov chain.

Theorem 1. Given bipartite marginals quantum states $\rho_{A B} \in \mathcal{L}\left(\mathcal{H}_{A B}\right), \rho_{B C} \in \mathcal{L}\left(\mathcal{H}_{B C}\right)$ compatible with a $Q M C$ state, say $\rho_{A B C}$, then the solution to the maximum entropy estimator is $\tilde{\rho}_{A B C}=\rho_{A B C}$, and moreover $\tilde{\rho}_{A B C}$ can be algebraically recovered via the Petz map, concretely:

$$
\tilde{\rho}_{A B C}=\rho_{B C}^{\frac{1}{2}} \rho_{B}^{-\frac{1}{2}} \rho_{A B} \rho_{B}^{-\frac{1}{2}} \rho_{B C}^{\frac{1}{2}}=\rho_{A B}^{\frac{1}{2}} \rho_{B}^{-\frac{1}{2}} \rho_{B C} \rho_{B}^{-\frac{1}{2}} \rho_{A B}^{\frac{1}{2}} .
$$

Next, we provide conditions for two bipartite marginal quantum states $\rho_{A B} \in \mathcal{L}\left(\mathcal{H}_{A B}\right), \rho_{B C} \in$ $\mathcal{L}\left(\mathcal{H}_{B C}\right)$ to be compatible with a QMC state .

Theorem 2. Two bipartite marginals quantum states $\rho_{A B}$ and $\rho_{B C}$ are compatible with a $Q M C$, say $\rho_{A B C}$, iff the operators $\varrho_{A B C}=\rho_{B C}^{\frac{1}{2}} \rho_{B}^{-\frac{1}{2}} \rho_{A B} \rho_{B}^{-\frac{1}{2}} \rho_{B C}^{\frac{1}{2}}$ and $\sigma_{A B C}=\rho_{A B}^{\frac{1}{2}} \rho_{B}^{-\frac{1}{2}} \rho_{B C} \rho_{B}^{-\frac{1}{2}} \rho_{A B}^{\frac{1}{2}}$ coincide $\varrho_{A B C}=\sigma_{A B C}$.

The Maximum Entropy Principle, in the form of Principle of Minimum Updating, is at the basis of the inferential updating of DOs [15]. This approach provides yet another perspective of Problem 1. Given a quantum system $\left\{X_{1}, \ldots, X_{n}\right\}$ on the separable Hilbert space $\otimes_{i} \mathcal{H}_{X_{i}}$, let $\varrho$ be our prior knowledge about the system. Assume that now we have access to a new set of information about the system provided as a family $\left\langle\Theta_{i}\right\rangle$ of expected values of the Hermitian operators $\left\{\Theta_{i}\right\}$. Then, the most unbiased posterior $\mathrm{DO} \rho$ corresponds to the one that minimises the relative entropy distance with the prior DO under the given constraints:

$$
\rho=\exp \left(\lambda \mathbb{I}+\sum_{i} \alpha_{i} \Theta_{i}+\ln \varrho\right),
$$

where the Lagrange multipliers $\left\{\lambda, \alpha_{i}\right\}$ are determined by the constraints $\operatorname{Tr}[\rho]=1$ and $\operatorname{Tr}\left[\rho \Theta_{i}\right]=\left\langle\Theta_{i}\right\rangle$. The process $\varrho \stackrel{\left\langle\Theta_{i}\right\rangle}{\longrightarrow} \rho$ is addressed as quantum Bayesian updating [15]. Problem 1 can be restated as the following 2-step quantum Bayesian updating process:

$$
\frac{\mathbb{I}}{d_{A B C}} \stackrel{\rho_{A B}}{\longrightarrow} \tilde{\rho}_{A B C}^{\prime} \stackrel{\rho_{B C}}{\longrightarrow} \tilde{\rho}_{A B C}
$$

where $\quad \tilde{\rho}_{A B C}^{\prime}=\rho_{A B} \otimes \frac{1}{d_{C}} \mathbb{I}_{C} \quad$ and $\quad \tilde{\rho}_{A B C}=\exp \left(\sum_{i j} \eta_{i j} \Lambda_{i}^{(B)} \Lambda_{j}^{(C)}+\log \tilde{\rho}_{A B C}^{\prime}\right)$.

The Lagrange multipliers $\left\{\eta_{i j}\right\}$ are determined by the constraints $\operatorname{Tr}_{A}\left[\rho_{A B C}\right]=\rho_{B C}$ and $\operatorname{Tr}\left[\rho_{A B C}\right]=1$. We observe that the solution of the 2-step Bayesian updating process does not necessary coincides with Equation (1), unless the two marginals are compatible with a tripartite QMC. In other words, Equation (5) strictly depends on the order of the data, while Equation (1) does not. A new characterisation of QMC at the light of quantum Bayesian updating is given in the following result.

Theorem 3. Given a couple of marginals $\rho_{A B}$ and $\rho_{B C}$, they are compatible with a tripartite $Q M C \tilde{\rho}_{A B C}$ iff the following diagram commutes

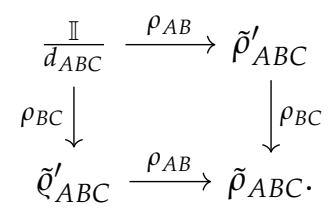


A trivial corollary of the above theorem is that the 2-step quantum Bayesian updating process coincides with Equation (1) iff the provided marginals are compatible with a QMC.

\section{Discussion}

Addressing the problem of inferring the tripartite DO that maximises the von Neumann entropy given two bipartite marginals, we find out that the classical results of algebraic recoverability can be extended to the set of DO addressed as quantum Markov chain.

We have shown that QMCs hold very interesting properties, namely they coincide with the quantum states for which a 2-step Bayesian update is commutative. Moreover, for QMC, we expect many classical learning/inference techniques to be easily adapted, including the efficient learning procedures for trees which is a particular case of the Quantum Hammersley-Clifford theorem by Poulin and Petz. For non QMC, we can still apply algebraic geometry algorithms, based on Tarski-Seidenberg theorem, to extract the optimal DO that maximises the Tsallis entropy (which approximates asymptotically the von Neumann entropy), but such methods are very inefficient.

A detailed, geometrical if possible, characterisation of the quantum states (1) is under exam, in particular to determine if a general tripartite maximum entropy DO is in some meaningful way close to a quantum Markov chain, in order to obtain an approximate algebraic recovery. The multipartite case, namely quantum Trees, is also under analysis.

Acknowledgments: This research was funded by Fundação para a Ciência e a Tecnologia (Portugal) through the grant number PD/BD/114332/2016 and FCT project PREDICT FEDER-029877.

Conflicts of Interest: The authors declare no conflict of interest.

\section{References}

1. Jaynes, E.T. Information Theory and Statistical Mechanics. Phys. Rev. 1957, 106, 620-630.

2. Cover, T.M.; Thomas, J.A. Entropy, Relative Entropy and Mutual Information. In Elements of Information Theory, 2nd ed.; Wiley: New Delhi , India, 2006; pp. 13-56.

3. Bishop, C.M. Graphical Models. In Pattern Recognition and Machine Learning; Springer: New York, NY, USA, 2006; pp. 359-418.

4. Chow, K.; Liu, N. Approximating Discrete Probability Distributions with Dependence Trees. IEEE Trans. Inf. Theory 1968 14, 462-467.

5. Sutter, D.; Fawzi, O.; Renner, R. Universal recovery map for approximate Markov chains. Proc. R. Soc. A 2017, 472, 2186.

6. Hayden, P.; Jozsa, R.; Petz. D.; Winter, A.; Structure of States Which Satisfy Strong Subadditivity of Quantum Entropy with Equality. Commun. Math. Phys. 2004, 246, 359-374.

7. Petz, D. Monotonicity of quantum relative entropy revisited. Rew. Math. Phys. 2003, 15, 79-91.

8. Fawzi, O.; Renner, R. Quantum Conditional Mutual Information and Approximate Markov Chains. Commun. Math. Phys. 2015, 340, 575-611.

9. Ibinson, B.; Linden, N.; Winter, A. Sufficient Robustness of Quantum Markov Chains. Commun. Math. Phys. 2008, 277, 289-304.

10. Sutter, D. Approximate quantum Markov chains. In Approximate Quantum Markov Chains; SpringerBriefs in Mathematical Physics; Springer: Cham, Switzerland, 2018; Volume 28.

11. Kim Isaac, H. Operator extension of strong subadditivity of entropy. J. Math. Phys. 2012, 53, 122204.

12. Ruskay, M.B. Remarks on on Kim's Strong Subadditivity Matrix Inequality: Extensions and Equality Conditions. arXiv 2012, arXiv:1211.0049.

13. Petz, D. Sufficient Subalgebras and the Relative Entropy of States of a von Neumann Algebra. Commun. Math. Phys. 1986, 105, 123-131. 
14. Lieb, E.H. Ruskai, M.B. Proof of the Strong Subadditivity of Quantum Mechanichal Entropy. J. Math. Phys. 1973, 14, 1938-1941.

15. Vanslette, K. The Quantum Bayes Rule and Generalization from the Quantum Maximum Entropy Method. J. Phys. Commun. 2018, 2, 2. 